\title{
Ética y calidad en salud: un binomio inseparable
}

Javier Luna Orosco E. ${ }^{1}$

Resumen: Interpretando que el principio del bien es el fundamento de la ética y que su giro aplicado no puede darse sin condiciones estructurales y funcionales de calidad, este artículo establece la relación indisoluble entre ética y calidad en el funcionamiento de los servicios médico sanitarios, y desarrolla además los condicionantes de calidad que hacen a su buena prestación y los instrumentos que permiten, en la práctica, medir y verificar su cumplimiento.

Palabras clave: ética, calidad, normas, indicadores de la calidad en los servicios de salud

\section{Ethics and health care quality: an inseparable pairing}

Abstract: By interpreting that beneficence principle is the ethical foundation and that its application is not possible without structural conditions and quality functions, this article establish the inseparable relation between ethics and quality in health care services functioning; furthermore, it develops quality determining factors for good benefits and instruments for verifying and measuring their practice and fulfillment.

Key words: ethics, quality, norms, quality indicators in health care services

\section{Ética e qualidade em saúde: um binômio inseparável}

Resumo: Interpretando o princípio do bem como o fundamento da ética e que sua expressáo aplicada não pode dar-se sem condiçóes estruturais e funcionais de qualidade, este artigo estabelece a relação indissolúvel entre ética e qualidade no funcionamento dos serviços médico-sanitários e desenvolve, ademais dos condicionantes de qualidade que fazem sua boa prestação e os instrumentos que permitem, na prática, medir e verificar seu cumprimento.

Palavras-chave: ética, qualidade, normas, indicadores da qualidade nos serviços de saúde

${ }^{1}$ Médico. Responsable del Área de Calidad y Auditoría en Salud, Ministerio de Salud y Deportes, Bolivia

Correspondencia: jloebiocal@hotmail.com 


\section{Introducción}

Sorprende pensar que, a pesar de los 5.000 años de transcurso histórico registrado en el quehacer de la salud y la medicina - acompańado constantemente por el desarrollo progresivo y creciente de lo que hoy se conoce como ciencia y tecnología-, tengamos la necesidad ineludible de seguir abordando problemas éticos cotidianos, los cuales, además de requerir un trato particularizado y no poder ser resueltos de manera general y definitiva, por su enorme diversidad y variabilidad, se hacen aún más complejos con aquellos derivados de problemas macroestructurales persistentes, o que tienden a profundizarse - dígase en lo social, económico, medio ambiental y hasta culturalen paralelo con otros de igual variabilidad cualitativa y cuantitativa, relacionados con los muchos desafíos e interrogantes que plantea el crecimiento demográfico humano y el acelerado desarrollo científico.

En buenas cuentas, material para la construcción y análisis especulativo de la "bioética paradojal simultánea" ${ }^{1}$, que funde en una sola, de múltiples matices, la "bioética cotidiana de situaciones persistentes" con la "bioética de frontera de situaciones emergentes", como las denomina y clasifica Berlinguer(1).

Esto requiere de un constante ejercicio de revisión, reflexión y adecuación, sin apartarse jamás

\footnotetext{
${ }^{1}$ Proponemos el concepto de "bioética paradojal simultánea" como un espacio de reflexión ética en el que coexisten situaciones contradictorias y polares en una misma realidad vivencial de tiempo y espacio, provocando profundas crisis individuales y colectivas que, si bien han estimulado transformaciones de variable importancia en prácticamente todos los campos del saber y la actividad humana, no encuentran hasta el presente su solución, pareciendo ser ésta, por el contrario, más lejana y compleja en la medida en que el mundo crece y la población mundial aumenta. Los ejemplos son numerosos, pero entre los más demostrativos están: la enorme brecha entre los extremos de riqueza y pobreza, cada una con sus propias consecuencias derivadas; la dependencia cada vez mayor de un ser humano condicionado inexorablemente a la tecnología de las máquinas que, en lugar de servirlo, lo somete y tiende a reemplazarlo, y la demencial inversión armamentista de un considerable número de países. Durante la realización del Primer Congreso Boliviano de Bioética, en 2001, propusimos la "bioética de intervención” como una bioética de movilización social para cambiar rumbos y hacer de ella lo que Potter quiso desde sus inicios, es decir, una ciencia de la sobrevivencia bajo la doctrina ética del bien, la justicia y la solidaridad humana, pero sobre la base de un trabajo constante que penetre la conciencia social y determine que todos los países del mundo la asuman como una razón de Estado permanente e inclaudicable.
}

de los imprescriptibles fundamentos de la ética, cuales son el bien y la justicia, como acertadamente los identifica $\operatorname{Rawls}(2)$, y que no es tarea fácil cuando la equiparamos con la que debe darse de manera simultánea a través de la asistencia médico sanitaria, cuyo carácter de realización pragmática y directa conlleva el primer gran deber ético de todo prestador de servicios de salud: el conocimiento preciso, permanente y actualizado de lo que hace, procurando hacerlo siempre bien sin provocar daño.

Todo ello nos lleva a relacionar lo ético, que en sentido estricto corresponde al campo de lo filosófico, con lo fáctico, como lo comprendió Thomas Percival cuando rescató la ética de lo puramente especulativo y dió lugar al primer código de ética aplicada al ejercicio de la medicina, a través de su obra "La ética médica como código de leyes y preceptos adecuados a la conducta profesional de médicos y cirujanos" $(3)^{2}$.

Con este importante antecedente surgirá más tarde el término "deontología" (de deonthos, deber, y logos, tratado) aplicado a la profesión médica por Bentham, definiéndolo - con un sentido restringido de "Tratado de los deberes y etiqueta profesional" - como "la ciencia que se ocupa de la determinación de los deberes que han de cumplirse si se quiere alcanzar el ideal del mayor placer posible para el mayor número posible de individuos"(3:102), e interpretado actualmente como el conjunto de reglas y normas para regular las relaciones humanas del equipo de salud con los pacientes y la sociedad en general, así como entre sus miembros, cumpliendo deberes sobre una base moral de conciencia, corrección y justicia. En buenas cuentas, sobre una base ética que no hace otra cosa que confirmar el sentido aplicado o utilitario que Percival le confirió a la ética, reflejado prácticamente en los códigos actuales que norman el ejercicio de las profesiones en salud, como es el caso del así llamado Código de Ética y Deontología Médica del Colegio Médico de Bolivia(4).

Sin embargo, el sustrato de la ética médica es mu-

\footnotetext{
2 "Medical Ethics or a Code of Institutes and Precepts adapted to the Professional Conduc of Physians and Surgeons".
} 
cho más consistente que el simple cumplimiento frío del deber con el conocimiento idóneo de lo que debe hacerse, porque además de incluir el paradigma de la autonomía, no maleficencia, beneficencia y justicia — que también puede aplicarse de manera fría y automatizada - agrega la propensión afectiva, conciencial, espiritual y volitiva de servir al paciente-persona en una relación humana paritaria, comprendiendo a plenitud la significación y trascendencia de su dignidad "como el atributo inalienable del ser humano para ser reconocido en su condición esencial de vida y racionalidad individual, única e irrepetible desde el principio hasta el fin de los tiempos"(5).

"Se deduce entonces que la relación de los pacientes con quienes los atienden es algo que depende de la formación individual y la valoración ética que tengan los prestatarios por sus actos, lo cual conlleva la necesidad de reflexionar profundamente sobre el tema, tanto más si la pureza del diálogo y las relaciones humanas pueden verse empañadas cuando, según la interpretación puramente jurídica, se entiende la prestación de un servicio como simple contrato entre partes. En este caso, lo material que entra en juego puede distorsionar las conductas, al punto de hacer pensar al paciente que debe ser curado porque paga o tiene seguro, y al médico que debe curarlo lo mejor posible porque percibe un salario o le pagan sus honorarios. Esta interpretación reduccionista y sesgada, que disminuye el carácter sublime del acto médico y de quienes lo realizan o contribuyen a su realización, está llevando a la medicina actual a una seria crisis, en la cual abundan los juicios y el temor e inseguridad de muchos profesionales que, con el fin de proteger su responsabilidad personal e institucional y hacer frente al riesgo de un potencial equívoco siempre latente, se ven obligados a utilizar recursos diagnósticos y terapéuticos muchas veces innecesarios, que, a más de elevar los costos de atención, pueden no ser inocuos o carentes de complicaciones" (6).

Con lo dicho estaríamos delimitando el complejo escenario ético en el cual se realiza el quehacer médico sanitario y sus determinantes, faltando sin embargo mencionar la relación indisoluble ética-calidad —motivo principal del presente escrito- - adelantándonos a decir que el cumplimiento cabal de la ética aplicada en la práctica de la salud y la medicina requiere de entornos estructurales y funcionales concordantes y propicios, que no pueden ser sino condiciones de calidad y seguridad. Es decir, un conjunto de características que permiten un quehacer bien hecho, ya sea relativo a servicios o productos, y que provoquen satisfacción en quienes lo reciben y en quienes lo realizan.

Por otra parte, si aceptamos que el servicio es la razón de ser de toda institución de oferta pública, cuyo cometido es atender y satisfacer los requerimientos de la población, no lograr que sus prestaciones sean confiables y aceptadas, o en su caso que sean cuestionadas, es un contrasentido a su propia existencia, concluyéndose que la calidad es la condición esencial o intrínseca de cualquier servicio, tanto más si nos referimos a los que tienen relación con los valores más preciados de la vida, la salud y el bienestar de las personas.

A continuación expondremos las condicionantes que se deben cumplir en el afán de lograr que la calidad sea una realidad, identificando sus indicadores y aplicando instrumentos expresamente diseñados que permitan verificarlos y medirlos, más allá de la pura elaboración teórica o el discurso.

\section{Condicionantes de la calidad}

No puede haber calidad sin ordenamiento y éste no existe si no se acompaña de un marco regulatorio claramente definido, difundido, conocido, asimilado y cumplido por todos. En la realidad, esto no ha sido debidamente comprendido en el sector salud, por la tendencia práctica y asistencialista predominante, que ha supuesto que su rol es la atención directa de los pacientes con la sola aplicación de conocimientos y experiencia, sin reparar en la necesidad de contar con un marco normativo que permita orientar su trabajo y cumplir sus prestaciones por un sendero de mayor seguridad y certidumbre. Tal situación le ha provocado mayor vulnerabilidad y desventajas frente a otros sectores del quehacer humano, que han hecho de la norma el primer requisito para cumplir sus funciones, como sucede con el sector 
jurídico o financiero. Sin embargo, la necesidad normativa estuvo siempre presente $y$, ante la falta de capacidad rectora de los niveles de mando supraestructurales, los niveles operativos tuvieron que definir sus propias normas de carácter muy específico o parcial, sin asumir la importancia de un contexto general sistémico que transversalice la aplicación de un marco normativo general, completo y único para todos los sectores que se ocupan de atender la salud de las personas, entendiéndose que todas gozan de los mismos derechos y merecen la misma calidad, independientemente de si la reciben en un seguro, un hospital público o una clínica privada.

Consecuencia de lo dicho fue la diversidad normativa que en mayor o menor medida sigue existiendo - especialmente en niveles institucionales autónomos que mal entienden la autonomía- y que en vez de orientar desorienta, requiriendo de una depuración que en los procesos de mejoramiento de la organización sanitaria tendría que darse, para visualizar mejor aquellas normas troncales que, al ser reconocidas y aceptadas por todos, sean jurídicamente válidas para su cumplimiento, permitiendo así que se haga realidad este principio de definición normativa, como hemos venido en llamar al primer condicionamiento de la calidad, dentro del ordenamiento planteado.

Por tales razones, resultan inaceptables las diferencias de calidad de atención entre establecimientos de salud que son pares por pertenecer a un mismo nivel de complejidad, para ofrecer servicios de salud similares y de igual capacidad resolutiva, situación que, sin embargo, se da en los hechos, porque las condiciones de ordenamiento y cumplimiento de normas son disímiles o porque varían las estructurales y de funcionamiento.

Ante esta última afirmación es que surge la necesidad de cumplir otro gran factor condicionante de la calidad, cual es la caracterización de los servicios y establecimientos de salud, entendiéndose por tal la descripción detallada de un modelo que establezca requisitos mínimos en cuanto a condiciones de calidad y seguridad de la infraestructura, equipamiento, dotación de recursos materiales, insumos y medicamentos, dotación cualitativa y cuantitativa de recursos humanos, flujos funcionales y, finalmente, normas de organización y funcionamiento, entre las cuales cobra singular importancia la claridad y especificidad de los manuales de funciones del personal, en el entendido que todos forman parte del equipo de salud con responsabilidades compartidas de acuerdo con dichas funciones. La caracterización es el perfil del servicio o el establecimiento de salud, resultando obviamente tan variado y diferente como variados y diferentes son los establecimientos o servicios de salud, considerando sus niveles de complejidad o el número de unidades especializadas y distintas que pudiesen poseer.

La caracterización es importante porque es el referente que permite adecuar los servicios y establecimientos de salud de acuerdo con su respectivo modelo que, como ya se dijo, regula en detalle las condiciones mínimas para ofrecer servicios de calidad y seguridad, de acuerdo con las finalidades propias. Por otra parte, determina que los entes gestores responsables de los servicios de salud cumplan con el aprovisionamiento y mantenimiento de los mismos, de acuerdo con la caracterización, puesto que de no hacerlo así, además de incumplir la norma, estarían negando las condiciones de calidad necesarias para su correcto funcionamiento, lo cual implica debilidad o vulnerabilidad institucional, lo que a su vez puede determinar vulnerabilidad de los pacientes por las deficiencias existentes, y vulnerabilidad de los prestadores que se desempeñan en condiciones de precariedad o desventaja.

De acuerdo con lo anterior, es fácil deducir la importancia jurídica de la caracterización, porque define las responsabilidades de los entes gestores en cuanto a dotar y mantener los servicios de salud no de manera aleatoria, sino de acuerdo con una norma que garantice condiciones de calidad y seguridad uniformes y equiparables en todos ellos.

Cabe agregar además que las condiciones de calidad llevan implícitas condiciones de seguridad, siendo el tema de seguridad en la infraestructura, equipamiento e instalaciones un importante rubro de prevención constante que se relaciona 
con los "hospitales seguros", fuertemente promocionados por la Organización Panamericana de la Salud OPS/OMS ante la posibilidad de circunstancias catastróficas eventuales o naturales.

Un componente complementario, pero no menos importante, se refiere a la sostenibilidad y mantenimiento de las condiciones de calidad en lo estructural, funcional, gerencial, administrativo y financiero, que muchas veces se constituye en el principal desafío cuando se instalan servicios. De ahí la seriedad con la que se debe planificar la construcción y habilitación de nuevos centros de salud, en cuanto a definir con precisión, justificada técnicamente, dónde asentarlos y cómo lograr el máximo beneficio en su rendimiento, evitando las dispersiones que implican mayor gasto en detrimento de otras necesidades de salud, saneamiento y bienestar humano.

\section{El triángulo de la calidad}

Sobre la base de la caracterización y de acuerdo con todo lo mencionado hasta aquí, el componente de la calidad tiene tres condiciones imprescindibles para la asistencia y otras tres igualmente imprescindibles para la gestión, siendo imposible el funcionamiento de cualquier servicio de salud si cualquiera de ellas falta. Para el caso de la asistencia, las tres condiciones son estructurales y se refieren a recursos humanos, recursos físicos de infraestructura y equipamiento, e insumos ma- teriales y fármacos; en tanto que para la gestión las condiciones son funcionales y tienen que ver con el ordenamiento basado en las normas, los flujos funcionales y la sostenibilidad. Tanto las condiciones estructurales como las funcionales conforman triángulos perfectos, es decir, equiláteros, como significando la proporción armónica de todas ellas, de tal manera que la insuficiencia de cualquiera desequilibra y altera el conjunto de su respectivo triángulo.

A su vez la sobreposición o ensamblaje de los dos triángulos, determina la aparición de otro triángulo, también perfecto o equilátero, que no es otra cosa que el triangulo de la calidad, simbolizando que la consubstanciación proporcional y armónica de todas las condiciones de calidad determina la existencia real de la calidad total que, por principio, no puede darse a medias. Es decir, la calidad como condición se da o no se da, resultando impropio referirse a ella como buena, mediocre o mala.

Según nuestro parecer, el gráfico 1 refleja bien lo explicado, agregando únicamente que la ubicación de los recursos humanos en el vértice superior del triángulo de asistencia, así como el ordenamiento basado en normas en el vértice homónimo del triángulo de gestión, han sido puestos a propósito para significar su importancia como vértices estratégicos, a partir de los cuales se desprende y se comanda la estructura general.

\section{Gráfico 1}

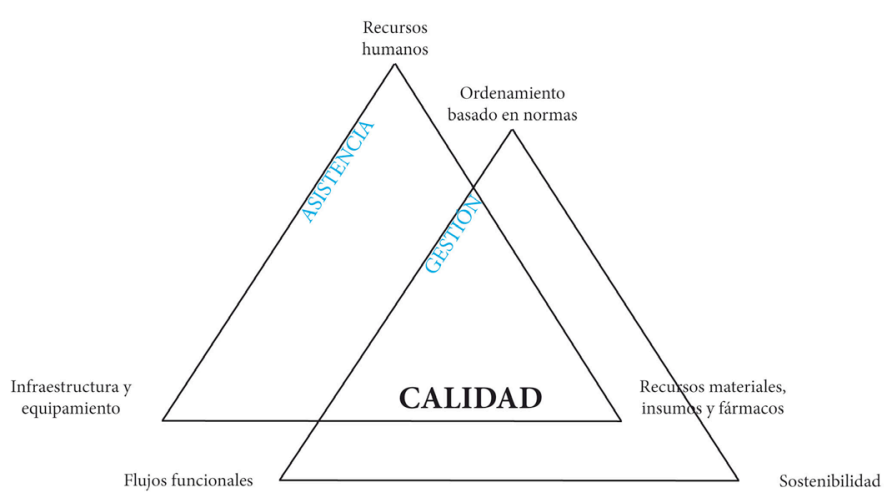

CARACTERIZACIÓN 


\section{Marco de competencias}

Una vez se tiene el ordenamiento basado en normas y la caracterización, un tercer componente fundamental - concordante con una organización tendiente a la centralización normativa y la descentralización operativa- es la precisión del marco de competencias de los grandes entes gestores institucionales, cuyo cometido es la conducción y mantenimiento de los establecimientos de salud desde el nivel nacional hasta el nivel local o territorial, pasando por los niveles departamentales (estatales o provinciales, según la configuración y división geopolítica de cada país) y los niveles municipales.

El marco de competencias está en función del cumplimiento de la caracterización, porque como fue dicho- define el rol de cada uno de los entes gestores en cuanto a dictación normativa, dotación de infraestructura, equipamiento y recursos materiales, dotación de recursos humanos y gestión de seguimiento, supervisión y control, componentes todos que, al tener sus específicos actores para su cabal cumplimiento de acuerdo con el marco de competencias, son identificados rápidamente cuando incumplen o mal cumplen dichas competencias, definiéndose ante la ley cuál es su responsabilidad y qué grado tiene.

El marco de competencias de tales actores no debe ser confundido con las funciones operativas que cumplen de manera directa y cotidiana los prestadores de los servicios de salud, contenidas como manuales de funciones y protocolos de atención en las respectivas normas de organización y funcionamiento que forman parte de la caracterización, y que también permiten asignar responsabilidades específicas.

Cabe agregar que, en el marco de competencias, la participación y gestión de los ciudadanos puede jugar un rol decisivo porque, al ser ellos los sujetos directos de las prestaciones que se otorgan en los servicios de salud -y por tanto interesados en la capacidad y calidad de la oferta y sus resultados-, podrían constituirse en los mejores vigilantes de los servicios a ellos dirigidos.

\section{Derechos y deberes}

Siguiendo la línea del ordenamiento y en el mismo afán de velar por la ética del comportamiento y promover la calidad, está la necesidad de definir con claridad la relación entre quienes reciben los servicios de salud y quienes los brindan; ambos en condición de usuarios, ya sea externos, como pacientes o clientes, o internos, como funcionarios profesionales, técnicos, auxiliares o administrativos.

Para ambos la observancia de derechos y deberes constituye la base sobre la cual se asienta una relación humana interactiva, que si bien en el pasado fue vertical y paternalista de los que otorgaban la atención "activamente" a quienes la recibían "pasivamente", hoy se ha transformado sustancialmente por el reconocimiento y cumplimiento universal del principio de autonomía o respeto a la voluntad del paciente, cuya expresión más sustantiva es la aplicación del consentimiento informado en un escenario vivencial muy diferente al de las demás actividades humanas, y que en determinadas circunstancias puede cobrar características dramáticas. De ahí la importancia del trato respetuoso y humanizado con el paciente y sus familiares desde el primer encuentro, "sabiendo que basta una mirada, una palabra o una actitud para que se incrementen o se pierdan los naturales temores y la confianza del paciente, considerado siempre como el aliado más fuerte en la lucha contra el mal que lo aqueja”.

Se comprende entonces, que el consentimiento informado no es una mera formalidad de un documento que se debe llenar, sino todo el proceso humano de acercamiento y convencimiento que, además de darse en las visitas médicas u otras circunstancias en que se aplican tratamientos o se realizan exámenes, también se produce en momentos aislados, a veces muy breves, que conceden posibilidades adicionales para conversar, detectar y resolver temores, transmitir mayor confianza e interés, y generar corrientes afectivas que repercuten favorablemente en los procesos de curación o rehabilitación. 
Tener presente el cumplimiento de derechos y deberes es entonces un tema clave, que vincula la ética del comportamiento con la calidad de los servicios de salud, razón por la cual se considera útil que, a manera de impresos en formato de cartel, pudiesen ser colocados visible y permanentemente en las salas de espera de hospitales $\mathrm{u}$ otros establecimientos menores(6).

\section{Instrumentos de medición de la calidad en Bo- livia}

Valorando y procurando asimilar el amplísimo basamento teórico de la calidad en salud - testimoniado a través de miles de páginas escritas en el mundo entero-, tenemos el convencimiento pleno que su aplicación práctica debe ser verificada y cuantificada aplicando instrumentos de comprobación y medición basados en indicadores de calidad generales y específicos, hasta lograr que cada unidad de servicios — con sus respectivos prestadores - sea capaz de identificar por sí misma cuáles deben ser "sus" propios indicadores de calidad en cada una de las prestaciones que brinda y que, a modo de guías, sean los referentes que orienten su rumbo de manera permanente. Este sería un ejercicio estimulante que promovería la superación constante hasta alcanzar la excelencia plena, que en buenas cuentas no es otra cosa que calidad posible de ser garantizada, expresión esta última muy comprometedora y que muchas veces se utiliza de manera libre y desaprensiva, sin comprender realmente su profundo significado ético y jurídico.

Con ese horizonte, el Área de Calidad y Auditoría en Salud del Ministerio de Salud y Deportes del Estado Plurinacional de Bolivia ha elaborado instrumentos normativos de calidad de acuerdo con su propia realidad, constituidos por manuales y guías de evaluación y acreditación de establecimientos de salud para los tres niveles de atención, según su complejidad y capacidad resolutiva; manual y norma técnica de auditoría en salud; manual de consentimiento informado; norma técnica para el manejo del expediente clínico y una guía básica de conducta médico-sanitaria, todos ellos en el marco de un documento de doctrina que establece las bases de organización y funcio- namiento del Programa Nacional de Calidad en Salud, en plena ejecución $(7,8)$.

De los instrumentos mencionados, los de evaluación-acreditación y auditoría han sido concebidos y diseñados no sólo para que los niveles de autoridad ejerzan su función normativa y de control externo -mediante las supervisiones y controles periódicos de los establecimientos y servicios de salud de acuerdo con su caracterización, determinando los correctivos necesarios según los resultados-, sino esencialmente para que los niveles operativos —allí donde se prestan los servicios directos a la población- sean capaces de autoevaluarse y auditarse a sí mismos, forjando un saludable juicio autocrítico que les permita corregir sus debilidades o errores con la oportunidad debida, siendo la prevención la visión ideal de la meta que se pretende.

\section{El gestor de la calidad}

Empero, todo este proceso no debería ser dejado al libre albedrío o buena voluntad de los que mejor se ordenen, sino que requiere de un animador y supervisor constante in situ, que no es otro que el gestor de la calidad en cada hospital o red de establecimientos, si se trata de centros menores, entendido como el elemento que da sustento al sistema, porque es aquel funcionario $-\mathrm{O}$ en su caso el equipo humano- con capacidad técnica, contratado expresamente para hacer el seguimiento y controlar que las distintas reparticiones o unidades de un hospital o de una red presten servicios de calidad constante de acuerdo con indicadores.

El gestor de la calidad — partiendo del diagnóstico de calidad del establecimiento, con un cronograma de supervisión y visita a todos sus departamentos, unidades y servicios- promueve el conocimiento y aplicación de los instrumentos de mejoramiento continuo y sostenido de la calidad, además de velar por el cumplimiento de las normas o directrices del propio establecimiento, de aquellas que emiten los comités de asesoramiento a la gestión y de las que emanan de la máxima autoridad ejecutiva, a la cual mantiene informada de manera constante sobre las condiciones de in- 
fraestructura, organización, equipamiento y dotación de insumos y medicamentos en las distintas unidades o servicios, en procura de solucionar oportunamente situaciones defectuosas o de incumplimiento.

Sumado a todo ello, el gestor de la calidad es quien recibe todas aquellas situaciones de reclamo o sugerencias relacionadas con la calidad de los servicios, procurando su solución inmediata $\mathrm{u}$ orientando el curso que deberían seguir si se trata de situaciones más graves o complejas, siendo además el recurso humano formado técnicamente para realizar auditorías médicas externas procedentes de otros ámbitos o para actuar como "par" con gestores de la calidad de otros hospitales, integrando los equipos hospitalarios que se organicen para el proceso de evaluación y acreditación periódica hospitalaria, de manera simultánea y cruzada.

\section{Principios éticos del sistema de salud}

El concepto de ética global sustentado por la UNESCO nos lleva inmediatamente a interpretar que la ética no sólo es una posibilidad humana, sino que también es posible hablar de una ética institucional y de una ética sistémica, entendiendo como sistema al "conjunto de cosas (entiéndase componentes) que, relacionadas entre sí ordenadamente, contribuyen a determinado objeto"(9), tanto más si existen normas únicas y generales que, al articularlas, las hace interdependientes, integradas y complementarias.

Dicho esto y aceptando que es propio hablar de una ética que se extiende al sistema, es posible identificar principios que, a partir de la estructura sistémica general, faciliten la inducción y penetración ética en todos sus componentes, siendo algunos de tales principios los clásicamente establecidos por la seguridad social, como la universalidad, solidaridad, justicia y equidad, accesibilidad, oportunidad, continuidad y sustentabilidad.

A estos se agregan la integralidad e intersectorialidad, concibiendo la salud y sus determinantes como una totalidad que conjuga persona, familia, comunidad, tierra y medio ambiente; la inter- culturalidad que respeta las diferencias humanas según "sentires", usos y costumbres, procurando en su caso la adecuación de los servicios de salud en los ámbitos en los que se dan esas realidades, con recuperación e integración de sus saberes médicos originarios y, finalmente, la participación social como el derecho y el deber que tienen las comunidades urbano rurales y las organizaciones sociales de participar de manera directa real y efectiva en la toma de decisiones para la planificación, ejecución, administración, seguimiento y control de las acciones de salud, saneamiento y bienestar social, sobre la base de la identificación y priorización de sus necesidades y problemas, mediante representaciones legítimas, organizadas y cabalmente conscientes de su tarea en aras del bien común.

\section{Conclusiones}

Con todo el análisis realizado, corresponde recién dar la definición más completa y globalizadora de la calidad en salud, diciendo que es la "condición de la organización sanitaria que, de acuerdo con caracterización y normativa claramente definidas de sus servicios dentro de un sistema, permite cumplir funciones bien realizadas y seguras que cubren satisfactoriamente las expectativas, requerimientos y necesidades de los usuarios, con recursos optimizados y equitativamente distribuidos".

Esta definición nos induce a comprender con absoluta claridad la necesidad de hacer el bien hecho bien, aunándose en esta frase, con aparente retruécano, la ética como concepto — con toda su trascendencia filosófica-, con la calidad como procedimiento — con toda su significación pragmática-, concluyendo que no puede existir efectivamente la primera sin aplicar la segunda.

Introducción

El artículo $8^{\circ}$ de la Declaración Universal sobre Bioética y Derechos Humanos de la UNESCO, aprobada en 2005, establece que "al aplicar y fomentar el conocimiento científico, la práctica médica y las tecnologías conexas, se debería tener en cuenta la vulnerabilidad humana. Los individuos y grupos especialmente vulnerables deberían 
ser protegidos y se debería respetar la integridad personal de dichos individuos"(1). Sin entrar en el término utilizado en potencial, "debería", que más bien debiera ser imperativo — "se debe" o, mejor, "debemos"(2)—, es claro que la protección de los individuos y grupos más vulnerables es una de las misiones principales de la bioética en su reflexión sobre los valores en la atención y en los sistemas de salud.

\section{Referencias}

1. Berlinguer G. Questioni di vita: etica, scienza, salute. Torino: Einaudi; 1991: 3-26.

2. Rawls J. Teoría de la justicia. México: Fondo de Cultura Económica; 2006: 36.

3. Gisberth Calabuig JA, Verdú Pascual FA. Moral Médica, bioética y deontología. Tratado de Medicina Legal y Toxicología. Barcelona: Editorial Masson; 2000: 103.

4. Colegio Médico de Bolivia. Estatuto Orgánico y Reglamentos del Colegio Médico de Bolivia. Tarija: Trinidad-Beni; 2008.

5. Luna Orosco EJ. Relación con los pacientes en el quehacer médico sanitario. Revista Boliviana de Bioética 2005; 1(1): 18.

6. Luna Orosco EJ. Guía Básica de Conducta Médico Sanitaria. Publicación No 66, Serie Documentos Técnico Normativos. La Paz: Ministerio de Salud y Deportes (MSD), Bolivia; 2008: 21.

7. Luna Orosco EJ, Cáceres Vega E. Bases para la Organización y Funcionamiento del Proyecto Nacional de Calidad en Salud PRONACS. Publicación No 56, Serie Documentos Técnico Normativos. La Paz: Ministerio de Salud y Deportes (MSD), Bolivia; 2008.

8. MSD, Bolivia. Serie Documentos Técnico Normativos, Nos 57-65.

9. Diccionario de la Lengua Española. Editorial Espasa Calpe. Vigésima Segunda Edición, 2001. Tomo II: 2073.

Recibido: 27 de julio de 2010

Aceptado: 13 de septiembre de 2010 